\title{
Gaining a better understanding of the extrusion process in fused filament fabrication 3D printing: a review
}

\author{
Bahaa Shaqour ${ }^{1,2}$ (D) $\cdot$ Mohammad Abuabiah $^{2}$ (D) Salameh Abdel-Fattah ${ }^{2} \cdot$ Adel Juaidi $^{2}$ (D) Ramez Abdallah $^{2}$ (D) \\ Waleed Abuzaina ${ }^{2} \cdot$ Mohammad Qarout $^{2} \cdot$ Bart Verleije $^{3} \cdot{\text { Paul } \operatorname{Cos}^{1}}^{1}$
}

Received: 30 November 2020 / Accepted: 15 March 2021 / Published online: 2 April 2021

(C) The Author(s) 2021

\begin{abstract}
Additive manufacturing is a promising tool that has proved its value in various applications. Among its technologies, the fused filament fabrication 3D printing technique stands out with its potential to serve a wide variety of applications, ranging from simple educational purposes to industrial and medical applications. However, as many materials and composites can be utilized for this technique, the processability of these materials can be a limiting factor for producing products with the required quality and properties. Over the past few years, many researchers have attempted to better understand the melt extrusion process during $3 \mathrm{D}$ printing. Moreover, other research groups have focused on optimizing the process by adjusting the process parameters. These attempts were conducted using different methods, including proposing analytical models, establishing numerical models, or experimental techniques. This review highlights the most relevant work from recent years on fused filament fabrication 3D printing and discusses the future perspectives of this $3 \mathrm{D}$ printing technology.
\end{abstract}

Keywords Fused deposition modeling $\cdot$ Fused filament fabrication $\cdot$ Experimental analysis $\cdot$ Simulation $\cdot$ Melt extrusion $\cdot$ 3D printing

\section{Introduction}

Over the past few years, additive manufacturing (AM) technologies have developed rapidly. With this technology, 3D structures are produced by laying off 2D layers sequentially along the vertical axis. Presently, AM is considered to be a rapidly growing area that has made great technological

\footnotetext{
Mohammad Abuabiah

m.abuabiah@najah.edu

Bahaa Shaqour

bahaa.shaqour@najah.edu

Salameh Abdel-Fattah

salabdel@najah.edu

Adel Juaidi

adel@najah.edu

Ramez Abdallah

ramezkhaldi@najah.edu

Waleed Abuzaina

w.abuzaina@najah.edu

Mohammad Qarout

engmqarout@gmail.com
}

progress since it was first invented by Hull [1]. Thereafter, several AM techniques using materials such as metals, polymers, and ceramics have evolved and have been developed by many researchers throughout the world.

There are different advantages of AM technologies over the traditional manufacturing processes, such as the time of designto-manufacturing is lower, waste materials are lower, flexibility

Bart Verleije

bart@voxdale.be

Paul Cos

paul.cos@uantwerpen.be

1 Laboratory for Microbiology, Parasitology and Hygiene (LMPH), Faculty of Pharmaceutical, Biomedical and Veterinary Sciences, University of Antwerp, Universiteitsplein 1, S.7, 2610 Antwerp, Belgium

2 Mechanical and Mechatronics Engineering Department, Faculty of Engineering \& Information Technology, An-Najah National University, P.O. Box 7, Nablus, Palestine

3 Voxdale, Bijkhoevelaan 32, 2110, Wijnegem, Belgium 
in produced designs [2], complexity in manufactured geometries [3-5], and ability to introduce internal structures without a notable increase in the cost or turnaround time [2]. Moreover, AM provides great promise in terms of sustainable lightweight construction and the fabrication of complex multi-functional material structures in a single processing phase [6-9].

One of the well-known AM technologies is Fused Deposition Modeling (FDM $\left.{ }^{\mathrm{TM}}\right)$. It was first proposed by Stratasys, and Scott Crump led the FDM ${ }^{\mathrm{TM}}$ technology development at Stratasys in 1989 [10]. This has evolved at doubledigit annual levels and has been used not only for research purposes but also in various important sectors such as engineering, science, quick prototyping, medicine, and industry [11]. For this technique, objects are produced by melting a thermoplastic polymer, to extrude it through a nozzle, and then depositing the melted material layer-by-layer onto a build plate and the previously printed layers to finally create a replica of the digital model. The robustness of FDM ${ }^{\mathrm{TM}}$ and the cost-competitive design were the key reasons for its tremendous success in the industry [11].

This technology was also known by the term Fused Filament Fabrication (FFF). This term spread in the field after the expiration of the Stratasys FDM ${ }^{\mathrm{TM}}$ patent, especially because the $\mathrm{FDM}^{\mathrm{TM}}$ term was registered as a trademark for Stratasys. Mainly led by the Reprap society, a wide variety of open-source software and hardware was readily available. This enabled this technology to become more affordable, and more accessible and user-friendly, which enabled anyone with a basic knowledge of computer aided design (CAD) to facilely use the FFF 3D printer. FFF has become favored in a variety of sectors, ranging from educational institutes and the medical sector to aircraft manufacturers and military corporations. The use of FFF has become a necessity throughout the phases of development, prototyping, visual aid, and presentations.

Over the past few years, crucial technical improvements have been achieved in FFF technology. These advancements can be divided into two categories: (1) process development, and (2) material development. Remarkable attention has been focused on the advancement of the speed of printing, the maximum print dimensions, and the maximum production rate. Additionally, FFF has been shown to have significant potential for 3D printing with different materials and composites, such as continuous and discontinuous fiber-reinforced polymers and nanoscale composites in many different applications, varying from small scale prototype to large scale industrial applications $[12,13]$. With this wide variety of thermoplastic materials and composites that can be printed requiring only a few upgrades and modifications to the printer itself, FFF 3D printing became one of the most widely used AM technologies.

Despite the significant improvements and the progress in FFF technologies, it is still highly empirical and requires calibration. Additionally, optimizing the printing process parameters is mostly done experimentally. Previous reviews have focused more on discussing published work that aimed to optimize the process parameters using the experimental approach. Chohan and Singh [14], Jaisingh et al. [15], Harris et al. [16], and Popescu et al. [17] reviewed numerous publications to gain a greater understanding of the dependency of the FFF process parameters and the printing material on the mechanical properties of the final part. They also investigated the impact of various process parameters (individually and combined) on the mechanical behavior of the specimen. The scientific community is working rigorously to develop models that can predict materials' behavior upon printing. Furthermore, many researchers are working on correlating the process parameters during $3 \mathrm{D}$ printing with the produced product in terms of functional properties, such as mechanical integrity, and appearance such as surface finish.

This review provides an overview of various studies that aimed to improve our understanding of the process of the FFF $3 \mathrm{D}$ printing method, by proposing models that can predict the printing behavior of a material based upon its properties, such as thermal and rheological. Moreover, we will specifically focus on the extrusion system in the FFF 3D printer. Firstly, the main principles that govern this system will be explained and discussed. Then, recently published work on the extrusion process and the relationship between the performance of this process with the quality of the produced objects will be discussed and outlined. Finally, the future aspects of this technology and the key topics that will further guide the development of this $3 \mathrm{D}$ printing technology will be discussed.

\section{The heart of the FFF 3D printer}

The extrusion system in the FFF 3D printer is a key player in this process. This part of the printer consists of several sections: (1) A motor for extruding the filament, (2) a barrel through which the filament flows without melting, (3) a heating block in which the filament is melted, and (4) the nozzle in which the cross-sectional area of the flowing material is changed from the filament to printed road size [18]. Those sections are illustrated in Fig. 1.

The part that initializes the extrusion process is one of the main components that ensure a continuous flow of material [18]. This is vital for assuring the best quality of the produced parts. In a conventional FFF $3 \mathrm{D}$ printer, a stepper motor, either with or without a step-down gear, is used. Subsequently, it is connected to a driving gear, which is in direct contact with the filament on the one side and an idler on the other side. The pressure caused by this configuration on the filament must be adjusted correctly. Excessive pressure and high extrusion speeds can cause grinding of the filament, which can occur when using brittle filaments. On the other hand, insufficient pressure can cause slipping between the filament and the driving gear, which will cause inconsistent material flow [19]. 
Fig. 1 Detailed construction of the extrusion system in an FFF $3 \mathrm{D}$ printer highlighting the main features required for proper material extrusion

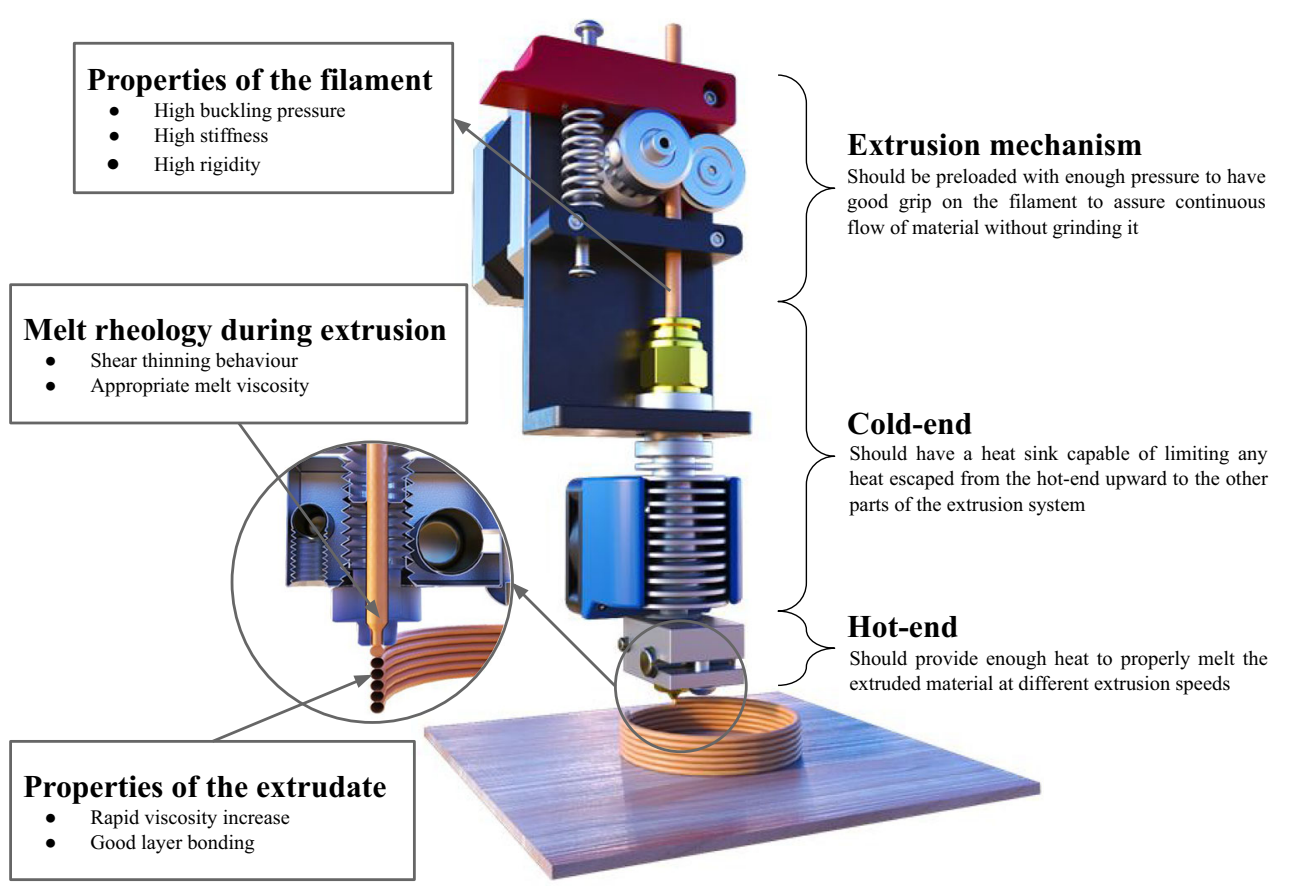

The material is then fed into a barrel, which typically functions as a barrier between the hot and the cold parts in the extrusion system and is mostly designed as a heat sink and is equipped with a fan. The optimum design of the barrel is crucial for preventing heat from escaping upward from the hot to the cold section [20]. However, if this was not prevented, it would then cause the filament to expand due to heat and it would get stuck inside the parallel, which will stop the material flow. Additionally, preserving the material in a nonmelted condition would aid with obtaining a successful extrusion process. This is due to the use of non-melted portion of filament as a piston to push the melted part through the nozzle $[21,22]$. However, the reduction in the stiffness of the nonmelted part, due to softening caused by excessive heating, will result in devastating effects on the continuity of the material flow.

The heating block is the part of the extruder in which the material is melted. In this 3D printing technology, thermoplastic polymers or composites are used [23]. These materials exhibit a change in their physical state upon heating above the glass transition temperature $\left(\mathrm{T}_{\mathrm{g}}\right)$ and this phenomenon is reversible [8]. This process is caused by the van der Waals weak forces and without the generation of new chemical bonding with the polymer matrix [24]. However, it is important to note that thermoplastics have very low heat transfer properties. Thus, the design of the heating block should assure sufficient heating of the material to enable that the required degree of material melting is reached. This can be achieved by increasing the length of the heating block to increase the material's residence time while under extrusion [25].
The final part of the extrusion system is the nozzle. This part is directly connected to the heating block as it should be heated at the same temperature. In this part, the cross-sectional area of the material is reduced. Normally, in typical FFF printers, the feedstock material is in the form of filaments with a cross-sectional diameter of 1.75 or $2.85 \mathrm{~mm}$. Thus, the nozzle has an input diameter that is similar to the one for the filament. However, the output of the nozzle varies with the diameter and ranges between 0.15 and $1.00 \mathrm{~mm}$ [26]. The nozzle output diameter has a direct effect on the accuracy and surface finish of the produced objects, as smaller diameters produce objects with fine details and high resolution. Nonetheless, it has a negative effect on the printing speed as a smaller diameter causes a lower material flow rate. Moreover, the nozzle's diameter size has a great effect on the extrusion pressure required for a continuous material flow. The maximum pressure that can be delivered by the extrusion mechanism should be taken into consideration when selecting the melting temperature $\left(\mathrm{T}_{\mathrm{m}}\right)$ and extrusion speed as they are the key players in the success of this part of the process [25].

To summarize the extrusion process, a thermoplastic polymeric material is fed in the form of a filament into the extrusion system by a stepper motor. This filament acts as a piston to assure a continuous flow of material. Then, the material is heated just above its melting point. This is very important to avoid over heating of the material which may cause unwanted oozing or over extrusion during non-printing and printing movements, respectively. Finally, the material exits from the nozzle into a smaller cross-sectional area. During this process, understanding the change in the material's melting and 
rheological behavior is crucial [27]. An ideal material should show high stiffness to assure optimum material feeding. Subsequently, this material should exhibit rapid changes in its viscosity with heating and during extrusion to minimize the required feeding pressure. Finally, this material should show rapid increase in viscosity upon extrusion to assure the preservation of the geometry of the $3 \mathrm{D}$ printed structure. Thus, thermoplastic polymers are widely used and act like a shearthinning material with excellent viscoelastic behavior upon heating and cooling [24]. The shear-thinning behavior describes a material with the reverse relationship between the viscosity and the shear rate. The viscoelastic behavior includes two main parameters which are the storage modulus and the loss modulus. When the storage modulus has a larger value than the loss modulus, the material becomes more solid-like, while when the loss modulus is larger, then the material acts more like a liquid-like one. After melting, the viscosity of the material decreases drastically. Moreover, during extrusion, the material is exposed to a high shear rate due to the decrease in the nozzle's diameter; this causes an even greater decrease in the viscosity of the material [28]. Finally and after extrusion, this high shear vanishes upon exiting the nozzle's output. Additionally, the material is quenched by the environmental temperature which is typically much lower than the nozzle's temperature. Thus, the material retains its high viscosity and solid-like behavior [27]. However, it is very important to have a sufficient time for proper diffusion between the deposited material and the previously printed layer. During the printing, the high temperature of the material being deposited acts as a heater that partially remelts the previous layer for a small portion of the time. This is key in order to achieve a temperature above $T_{g}$ and the crystalline melting temperature [29]. This condition initiates the welding and polymer fusion process between the two layers. This thermally driven process is called reptation, which is a model describing the polymer chains when melted as if moving within a tube that represents the topological constraints imposed by entanglements with other chains [30]. This process is highly affected by melt anisotropy and the developed crystal morphology. Thus, the non-isothermal process that occurs during $3 \mathrm{D}$ printing affects greatly the polymer's solidification behavior. This can be seen in non-crystalline polymer melts cooling below $\mathrm{T}_{\mathrm{g}}$ or semi-crystalline ones that nucleates and crystallize between $T_{m}$ and $T_{g}$ [31]. The crystallization behavior in semi-crystalline polymer has a great effect on layers deformation during printing. This is due to the effect of the crystallization process which induces dimensional variations [32]. A possible solution to reduce this effect is by the addition of fillers materials that slow down the crystallization process as can be seen in a work done by Fitzharris et al. [33]. Moreover, all the previously mentioned aspects play a vital role in improving the mechanical properties of the $3 \mathrm{D}$ printed objects.

\section{Gaining a better understanding of the extrusion process in the FFF 3D printer}

As many research groups have investigated the phenomena that are related to the extrusion process during 3D printing, this section will divide their work into two categories: (1) previous work that discussed the extrusion process inside the extruder, and (2) previous work that discussed the material behavior after being extruded and during the construction of the $3 \mathrm{D}$ printed part. Additionally, the included articles in this review were summarized in the supplementary table ST1.

\subsection{Previous work discussing the extrusion process inside the extruder}

There has been a great interest in understanding the extrusion process in FFF 3D printing since the spread of commercial machines that were provided by Stratasys. An example of such research can be seen in several articles published in the early 2000s. Bellini et al. [34] analyzed the response of the extrusion system to better understand its behavior, which would enable the design of a control system to control the material flow. After deriving an analytical model, they established a model based on dynamic systems modeling to study the response of the system based on a defined input. A comparison was made between the results obtained from this model and the experimental data. It was concluded that the slippage between the filament and the extruder's rollers caused the steady-state error in the system. On the other hand, the limitation in the motor torque, power, and the temperature variation in a liquefier, which directly affect the viscosity of the polymer, is considered to be the cause for the time delay in the response.

Moreover, Ramanath et al. [35] numerically analyzed the velocity gradient, pressure drop, and thermal behavior and compared these results with previously published analytical models. The polymer under investigation in this study was polycaprolactone (PCL), with an intended application of producing scaffolds for biomedical applications. Based on their results, it was found that the liquefier's temperature and geometry have a direct effect on the extrusion process. The material was melted after passing $34 \%$ of the total liquefier length. Additionally, Mostafa et al. [36] conducted a numerical and experimental analysis of Acrylonitrile butadiene styrene (ABS) loaded with iron particles. The numerical simulation was conducted on computational fluid dynamics (CFD) software with a focus on main parameters such as temperature, pressure drop, and flow velocity.

Moreover, Monzón et al. [37] considered the possibility of extrusion using fine nozzle diameters (i.e., $0.05 \mathrm{~mm}$ ). They used ABS as a testing material. Their work included establishing an analytical model and experimental analysis focusing on swelling. They used a conventional FFF printer to extrapolate 
the relevant conclusions when using fine nozzles. The nozzle and the envelope temperature were shown to be a key contributor to the die swelling; however, the nozzle temperature demonstrated a more significant effect. Additionally, it was found that there was a temperature variation along the nozzle, with a lower temperature found at the nozzle exit. This was caused by the nozzle's design and the location of the heating element. A swelling diameter factor, which represents the ratio between the nozzle's diameter and extrudate's diameter after the extrusion process, was proposed for this extrapolation. This factor was estimated to be equal to 1.249 . The calculations showed that using such a fine nozzle decreases the volume of flow by 215 times when compared with a conventional system; (i.e., $0.46 \mathrm{~mm}$ ) diameter.

After the expiration of the Stratasys FDM ${ }^{\mathrm{TM}}$ patent in 2010, open-source 3D printers started to be used by researchers and many publications on these systems arose [19]. This greatly benefited from the work done by Bowyer's and his colleagues, which developed into a global open-source society called the RepRap project [38]. The relatively cheap hardware and open-source software enabled researchers to contribute more to the development of the $3 \mathrm{D}$ printing process. This was limited using the commercial FDM ${ }^{\mathrm{TM}} 3 \mathrm{D}$ printers provided by Stratasys. Ortega et al. [39] designed a special nozzle equipped with a temperature and pressure sensor to investigate the process parameter and their effects on the extrudate swell and shape. They found that a higher swell was caused by a higher shear rate. This is because of the material's short residence time inside the nozzle. Additionally, they provided CFD results for the process, which corroborated the experimental data.

Anderegg et al. [40] redesigned the nozzle segment to equip it with a pressure sensor and double the temperature sensors along the liquefier. Adding the pressure sensor was very useful for understanding the relationship between the pressure inside the liquefier with time. The experimental data recorded showed a sigmoidal relationship between the pressure and time at the beginning of extrusion, while an exponential decaying one at the end of the extrusion. Additionally, a lower shear rate was observed at the beginning of the extrusion process which could negatively affect shear sensitive material such as ones reinforced with fibers. Moreover, it enabled a comparison between the experimental measurements and calculated values from previously developed analytical models. This comparison showed a $27 \%$ deviation which was caused by limitations in the parameters of these models such as nozzle geometry, isothermal and steady-state assumptions among others. Another important observation was the fluctuation in both temperature and pressure while the system was in an idle state. The fluctuation in the temperature was caused by the control system, which regulates it. This is typically a proportional-integral-derivative(PID) controller and the selection of proper parameters plays a vital role in lowering these fluctuations. On the other hand, the fluctuation in pressure was caused by temperature fluctuations as the material expands during the heating cycles.

Tlegenov et al. [41] introduced a method for detecting the nozzle clogging using a vibration sensor. For this purpose, an acceleration sensor was mounted on the extruder that has a fixed position while the printing platform has the ability to move in three axes. In this study, two types of extruders were used: direct and Bowden extrusion systems. Moreover, ABS, Polylactic acid (PLA), and flexible filaments, which demonstrate different mechanical properties, were used to examine the efficiency of each extrusion system. It was found that ABS was less sensitive to the nozzle's temperature and thus showed less clogging during extrusion than other filaments. Additionally, the Bowden configuration showed a greater likelihood of clogging when compared with the direct extrusion configuration.

Serdeczny et al. [25] designed an experimental setup that mainly depends on measuring the relationship between the feeding force and input filament speed at various nozzle temperatures. This group used the experimental data collected from their setup to validate an analytical model that was based on heat balance inside the barrel section of the hot end and independent of the pressure drop. They found that the relationship between the feeding force and feeding rate increased linearly and was highly dependent on the liquefier temperature, which was a limiting factor in this case. They noted that the limitation of the slow heat transfer within the hot end barrel can be solved by increasing its length to allow sufficient time for heat to properly melt the extruded material. On the other hand, they studied the relationship between the nozzle diameter and the swell ratio. From their experimental data, it was shown that there is a positive relationship between these two parameters. Additionally, they found that the swell ratio is positively affected by the flow rate and the liquefier temperature.

Peng et al. [42] investigated the extrusion process inside the hot end using two approaches. The first approach used a specially prepared polycarbonate (PC) filament with horizontally colored pigments along the filament to study the flow profile during extrusion. The second approach used a temperature sensor embedded inside the filament. This sensor enabled researchers to study the temperature changes that occur to the filament during the extrusion process. It was found that increasing the extrusion speed causes an increased deviation from an ideal isothermal flow. Thus, the experimental results suggest that the extrusion process is a highly non-isothermal process, especially at high extrusion temperatures. Moreover, it was concluded that the temperature at the center of the filament is lower than the inner walls of the hot end barrel. A blunted velocity profile was detected when the shape of the colored pigment was visualized 
after extrusion, which indicated a lower shear rate at the center of the filament compared to the outer surface.

Shadvar et al. [43] mainly focused on the swelling effect during extrusion, both numerically and experimentally. An extrudate was immediately quenched after extrusion to study the effect of extrusion temperature and speed on the swelling ratio. They found that the simulation resulted in a $20 \%$ increase in the values when compared with the numerical simulation. Such differences in the results were due to assumptions in the numerical model, temperature variation during the process, and also inaccuracies produced during the quenching process. The study authors concluded that the swell was mainly caused by the increased shear due to the change in the area primarily in the conical section of the nozzle.

Jerez-Mesa et al. [44] designed and ran finite element (FE) modeling to investigate the thermal performance of a RepRap $3 \mathrm{D}$ printer liquefier that depends on the airflow velocity introduced by the refrigerating fan. The airflow velocity, resulting from the fan, can be written as a numerical value and assigned as a percentage in the software. The result showed that the final achieved temperature at the top of the liquefier was influenced by the convection caused by heat dissipation. Therefore, this study suggested that the refrigerating fan must not be left out when extruding PLA. This influence was noticeable when a PLA material was extruded at $210^{\circ} \mathrm{C}$ while a cooling fan was set at $30 \%$ of its power. This showed a relevant influence at the top of the liquefier by reducing the temperature to $31.1^{\circ} \mathrm{C}$.

Other research groups have worked on developing models to better understand flow behavior inside the extruder and the nozzle. Yang et al. [45] provided a numerical model to investigate the extrusion process of fiber-reinforced polymers. Fibers were simulated using discrete element method particles, while polymer melt was simulated as a Newtonian incompressible fluid. Additionally, a physical model was presented to compute the drag force acting on the fiber and its reaction force returned to the surrounding polymer melt. However, heat transfer and energy equations were not included in this model. To test their model, two scenarios were analyzed: (1) ABS loaded with short glass fibers, and (2) Polyamide (PA) loaded with a continuous carbon fiber located in the center. Based on the proposed model and for the first scenario, during extrusion, the fibers are randomly located in the center of the liquefier, while they are more aligned and parallel near the walls. However, subsequently the extrusion fibers are randomly oriented due to hitting the printing bed. In the second scenario, the continuous fiber stays in the center due to the symmetry of the nozzle. However, after extrusion a large shift in the fiber occurs in the opposite direction of the movement of the extrusion head due to asymmetry of the extrusion conditions.

Moreover, Heller et al. [46] worked on simulating the fluid flow of fiber-reinforced polymers. They attempted to study the fibers' orientation during and after extrusion. For this work, two models were used; the fluid was modeled using incompressible stokes flow which is based on the NavierStokes equation with the inertial term being neglected, the fibers were simulated by modeling orientation diffusion and fourth-order orientation tensor in which a closure approximation was achieved. In this study, the material's behavior was analyzed both within the nozzle and also after extrusion. Thus, it could be concluded that the extrudate swell had a large effect on the fiber orientation, which will consequently affect the mechanical properties of the produced prints.

Mendes et al. [47] used a different approach for simulating the extrusion process inside the nozzle. In their work, microfluids were used to replicate the process of polymer melt extrusion. For this purpose and to assure that their method behaves similarly to the polymer extrusion, the Deborah (De) number, which is a dimensionless number used for studying the properties of fluids under specific flow conditions, and Reynolds number ( $\mathrm{Re})$, which is a dimensionless number used to predict the flow patterns in different flow situations, were monitored to assure they are as close as possible to the ones produced during extrusion. The study authors concluded that the De and Re numbers are key players in the material's flow behavior. It was found that at small Re and De, a Newtonian like fluid was observed with no instabilities or vortices formation. When the Re and De were increased, a change in the behavior was observed as some vortices started to appear. Higher Re and De caused larger vortices. The generated vortices were an indicator of un-extruded material near the walls of the nozzle. This phenomenon causes backflow behavior during polymer extrusion. The increase in this behavior may lead to material escape upward between the filament and barrel walls number.

\subsection{Previous work discussing the material behavior after being extruded and during the construction of the 3D printed part}

Since the beginning of the spread of commercial FFF 3D printers, researchers have worked intensively to simulate the $3 \mathrm{D}$ printing process. Some researchers focused on developing models that simulate the layer-by-layer printing process and the effect of the process parameters on the properties of the produced objects in terms of geometric accuracy and also mechanical performance. Li et al. [48] presented a theoretical and experimental analysis for predicting the elastic constant properties in an FFF system using ABS material. They also determined the effective stiffness, which is an average measure of the stiffness of the material that could be used for obtaining the required stiffness properties of the manufactured part. They found that a larger variety of laminates may be created by considering a different combination of raster angles in progressive layers. The minimum modulus of elasticity can 
be obtained by having a laminate with raster angles of (45/ $-45)$. Finally, the highest Young's modulus can be obtained in the laminate with raster angles of $(0 / 90)$. Moreover, Zhang and Chou [49] prepared a FE analysis model using element activations to simulate the mechanical and thermal phenomena in the FFF system. The model has also been used for residual stress and part distortion simulations to study the toolpath effects on the process. The simulation result shows that the short-raster tool-path causes higher residual stress, and thus possibly larger distortions than the long-raster and alternate-raster patterns (where both have similar stress distortion and distributions features). The long raster tool-path shows a stress concentration pattern at the bottom of the surface and each layer, the stress begins to accumulate at the initial deposition locations. Furthermore, the boundary condition can cause greater thermal gradients at the tool-path turning points and leave a noticeable stress accumulation mark. Finally, the measured data from the prototype experiments showed that the part distortion center has been shifted due to different tool-path patterns, which is in agreement with the simulation characteristics of the residual stress.

Bellehumeur et al. [50] investigated, analytically, and experimentally, the bond formation among extruded ABS filaments in the FFF process. The effects of some process parameters, such as extrusion envelope temperatures and dimensions of the extruded filaments were evaluated using a polymer sintering model. It was shown that the neck growth of the bonding zone was significantly affected more by the extrusion temperature than by the envelope temperature. At high temperatures, the extruded filament cannot maintain the complete bonding between the filaments in the current process. Finally, it can be concluded that the values for relative bond strength factors varied with the gap size between filaments. These simulation results, obtained from the model, showed a proper agreement with the experimental results. On the other hand, Costa et al. [51] worked on an analytical model to study the transient heat transfer of an extruded filament, taking into consideration the interaction with previously deposited material or the built platform. The rate of cooling decreases as more layers are deposited. It was found that the characteristics of the contacts between the extrudate environments play a major role in the temperature field and consequently on the bonding between layers.

During the past five years, there has been even more interest in producing models that can help simulate and predict the $3 \mathrm{D}$ printing process. Xia et al. [52] made a numerical model to study the fused deposition modeling 3D printing process which included the effect of different process parameters. Their model was based on the front-tracking/finite volume method which was established for simulating multiphase flows. This model enabled the visualization of the 3D printing process of different structures, such as two-layered cubes. This allowed the investigation of the characteristics of printed structures, such as the contact area between two deposited beads, in consecutive layers. Moreover, quantitative data could be gathered from the model and the effects of temperature gradient and deposited bead dimensions with different process parameters, such as printing speed and nozzle temperature, could be determined.

Zhang et al. [53] developed a numerical model to study the effect of process parameters on temperature variation during $3 \mathrm{D}$ printing. This model included many factors such as nozzle, bed and environmental temperatures, layer thickness, printing speed, and print dimensions and resolution. Based on their proposed model, it was found that the nozzle and bed temperature are important factors for determining the temperature variation during 3D printing. Additionally, the layer thickness or printing speed is inversely proportional to the cooling rate. Those parameters can be utilized to control the temperature variation of the printed object, which ultimately can improve inter-layer adhesion and the mechanical properties. For highresolution FFF 3D printers, the temperature variation is a key element in the printed object dimensional accuracy. Thus, proper and accurate control of the nozzle, bed, and environmental temperature is very important for a successful process.

Moreover, Liu et al. [54] created a numerical model that was based on the OpenFOAM CFD solver. This model was mainly utilized to simulate the deposition process while focusing on the effect of printing speed, printing temperature, and nozzle shape on the printed part quality. The authors found that the nozzle geometry has a significant influence on print quality; e.g., a rectangle nozzle with thick walls provides good quality compared with other shapes. They also noted that a lower printing temperature improves the final printing quality as the material will solidify faster. Furthermore, adding a pause between layers to stop the material flow will improve the printing quality, and materials with higher relaxation time have higher die swell. On the contrary, printing at low speed will have a bad effect on the printing quality and causes a pileup and local buckle. Finally, the authors used experimental data that was collected from Quinzani et al. [55] to validate their model which showed small differences compared with the obtained simulation results.

Xia et al. [56] made a numerical model to simulate the construction of three objects (bridge, inverted cone, and a rectangle) formed by parallel filaments using the FFF process. The simulation result demonstrates that the object constructed utilizing a material with low viscosity will have a slight fluctuation and become unstable (cone case). While, on the contrary, constructing an object with high viscosity will always rise and be stable. Furthermore, the heat losses are about twice lower for the object with high viscosity than for the one with low viscosity. The study authors also found that decreasing the spacing of the filament will lead to stronger squeezing with a large reheated area and larger deformation. Finally, the 
simulation result showed that a higher injection temperature will cause more deformation (bridge case).

Additionally, Bakrani Balani et al. [57] conducted an experimental, analytical, and numerical simulation to study the effects of printing parameters on the stability of deposited beads of PLA. This study showed that with a small nozzle diameter, a maximum value for shear rate can be obtained at the internal wall at a high inlet temperature. At the same time, decreasing the viscosity will enhance the adhesion between the deposited beads and layers, and a low viscosity will have a low precision result. Additionally, a multi-physics two-phase flow model was made to calculate the viscosity of the polymer and shear rate according to various inlet velocities and had been validated by an experimental setup. The results showed that the output material extrudates underwent severe deformation, caused by the 'sharkskin' effect when the shear rate is higher than $4000 \mathrm{~s}^{-1}$.

El Moumen et al. [58] carried out their experimental tests using a mini-single screw extruder fitted with a nozzle, to investigate temperature and residual stress fields during $3 \mathrm{D}$ printing of composite polymer, while a numerical model was created to simulate the FFF 3D printing process. They illustrated that the difference in temperature between the numerical simulation and experiments was less than $5 \%$. The temperature was determined at various zones (through the thickness and along the length) to predict the potential part distortion, stress concentration, and residual stress. This was conducted using two different printing approaches; (1)layerby-layer deposit printing process, and (2)line-by-line process filament deposit. The authors observed that the maximum stress was between the first and the second layers and decreased gradually with the composite thickness and the highest temperature gradient was recorded during filament deposition. They also found that the stress magnitude increased with printing time, which was induced by the decrease of the temperature and the solidification of the part. During the cooling phase, the von Mises stress reaches its maximal value; it takes $55 \mathrm{MPa}$ for the filament deposition process and $65 \mathrm{MPa}$ for the layer deposition process. An important gradient in the temperature was observed throughout the composite thickness and the stress concentration was higher when the temperatures of the printed part varied rapidly, and this stress can lead to delamination between the layers of the printed part.

Some studies focused on simulating the bond formation either between adjacent deposited beads or between layers as this is one of the key players in the mechanical properties. Costa et al. [59] presented an analytical solution to the transient heat conduction that takes place during filament deposition in fused deposition techniques by taking into consideration the deposition sequence. The computation of adhesion quality between adjacent deposited material segments has been also proposed. The resulting computation considered the main process parameters, such as filament dimensions and material, environment temperature, extrusion velocity, and sequence of deposition to predict the adhesion and the evolution of temperature during the deposition process and until cooling is completed. This study showed that insufficient adhesion between filament segments was anticipated at the lateral bottom regions of the produced part, and this was probably due to the more efficient heat conduction at this location with the support and environment. The study authors also found that $7 \%$ of the entire volume of the part will have poor adhesion by reducing the environment temperature from $70^{\circ} \mathrm{C}$ to $50^{\circ} \mathrm{C}$, whereas reducing the environment temperature to $40^{\circ} \mathrm{C}$ will increase this value to $52 \%$.

Moreover, Coogan and Kazmer [60] presented a simulation model using a diffusion-controlled healing technique for predicting the material bond strength between layers in the FFF process. The developed simulation model may be utilized to calculate the layer-to-layer strength of produced parts as a function of print settings and material properties. The results show that the simulated bond strengths can predict the measured bond strengths with a 0.795 coefficient of determination. The results indicate that the nozzle temperatures, larger fiber width, faster print speeds, and higher platform can produce greater bond strengths, and this is because each of these parameters allow for more polymer chain diffusion across the bond interface. The authors also found that the total diffusion reaches equilibrium value and begins to plateau as the interface temperature approaches transition temperature $(\mathrm{Tg})$.

Fonseca et al. [61] studied the bond connection between layers such as interlaminar strength and toughness of the components created by an FFF process. To do that, a set of experimental tests were performed followed by numerical analysis with pure and short fiber reinforced PA. More precisely, three experimental results were obtained for the following material, PA12 and PA12 loaded with carbon fibers. The results show that these materials have a quite a low cohesive strength and Young's modulus in comparison with traditional composites. These materials have a potential for application, especially when interlaminar fracture behavior loading is a crucial design parameter that needs to be considered.

Kallel et al. [62] used an experimental setup to study the bonding formation between the PLA filaments. The authors found that printing parameters like nozzle temperature, platform temperature, and feed rate can differentially influence the neck growth of filaments. They found that there is a high variation difference between the temperature of the filament and the setpoint, and this is increasingly influenced by the previously mentioned printing parameters. A coalescence test has been done to observe the neck growth evolution with temperature and time, and it appears that there are limits for reproducing the same conditions as observed during the process. The analysis shows a cyclic evolution with different temperatures between layers. Finally, a predictive model was proposed to predict the neck growth. However, the results 
showed a lower amount of neck growth than the experimental data, which can be due to the consideration that the authors did by choosing the constant heat transfer coefficient, heat capacity, the pressure of the nozzle, and polymer relaxation time.

The crystallization behavior has a great effect on the output from the 3D printing process, thus, many researchers focused on studying how semi-crystalline materials behave during printing. Northcutt et al. [63] work combined infrared (IR) thermography and Raman spectroscopy to show the effect of process conditions on the crystallinity of PCL. The testing setup consists of a 3D printer's extruder extruding on a moving belt. The extrudate then passes by a Raman spectroscope and IR sensor. The Raman spectra and the IR intensity were used to calculate the crystallinity as a function of distance or time from the nozzle. Using this configuration, the researchers could study the effect of the nozzle's temperature $\left(90-140^{\circ} \mathrm{C}\right)$ and flow rate $(1.8-3 \mathrm{~mm} / \mathrm{s})$ on the enhancement of the crystallization kinetics. Based on their results, it was found that the process conditions have a direct effect on the crystallization of the used polymer, when printing at a lower temperature with enhanced crystallization kinetics at a higher shear rate. IR measurements showed a fast cooling rate with the independence of the filament feeding rate.

Mcllroy and Graham [31] set up a numerical model to study the crystallization kinetics during non-isothermal melt extrusion-based 3D printing. The simulated results were validated by Raman spectroscopy measurements produced by Northcutt et al. [63]. The results from their study show an enhanced crystallization behavior due to flow-enhanced nucleation. Additionally, the crystallization time is improved at the surface of the deposited material, while, the inner section showed slower kinetics. The polymer stretch caused by the extrusion flow results in an inhomogeneous spherulites-size distribution and reduction in crystallization time at the surface. This is limited to low printing temperatures. On the other hand, the inner part of the deposited material has a quiescent kinetics and slower crystallization time. This is due to the big variation in the number of nuclei when compared with the surface. The researcher suggests that the flow-enhanced crystallization on the surface plays an important role in improving the mechanical strength of interface between each two consecutive printed beads. This is due to the formation of more spherulites which generate tie-chains across the weld interface.

Seppala and Migler [29] studied the temperature profile of the extrudate using an IR imaging sensor. ABS was used as a model polymer for this research. The main focus of this study is to investigate the spatial area directly around the active printing area and more specifically to study the welding behavior of the successive layers. Their results suggest that the extrudate cooling rate reaches $100^{\circ} \mathrm{C} / \mathrm{s}$ and stays above $\mathrm{T}_{\mathrm{g}}$ for around one second. Thus, only a small amount of heat is transferred to the layer below the current one being printed.
The time allowed for weld formation in this process was around two seconds. Moreover, the formed weld between the two layers does not go through the annealing process as the second layer below the one being printed never reaches $\mathrm{T}_{\mathrm{g}}$.

Moreover, some researchers used advanced analytical tools in order to have a better observation of the thermal history of the 3D printing process. Vaes et al. [64] used an IR sensor to study the temperature variation during the melted bead deposition while $3 \mathrm{D}$ printing. Afterward, the measured cooling and heating rates were used as an input for setting up a testing method to be used in a scanning chip calorimetry instrument. The researchers focused on understanding the crystallinity of a semi crystalline polymer after 3D printing. Two molecular weights of PA polymer were used in this test. Via this approach, the crystallinity of the tested PA was analyzed. The results of their study show that the nozzle temperature and the printing speed have a small effect on the crystallinity, while the build plate and the environment temperature have a more pronounced effect. Moreover, the lower molecular weight the more enhanced the crystallinity. Another important phenomenon that was heavily investigated is the layer distortion or the printed parts' warpage. This has a large effect on the final product's dimensional accuracy. Additionally, in some cases, it might cause a failure during the $3 \mathrm{D}$ printing process. Xinhua et al. [65] created a theoretical model to investigate the distortion mechanism in a PLA thin-plate part using the FFF 3D printing process. The model has been validated by experimental data using a 3D scanner to scan the final printed part. From the model and the experimental data, the result shows that the distortion levels decrease with latter layers in the printed part and the biggest deflection occurs at the four corners of the PLA thin plate. They also found that the distortion will increase by decreasing the layer thickness. A fast filling speed will reduce the distortion up to a limit after which it causes noise and violent vibration. Finally, the distortion can be reduced by having a lower nozzle temperature, and this is due to the lower temperature gradient.

Terekhina et al. [66] examined the effect of build orientation on the flexural quasi-static fatigue behavior. The main material studied in this work was PA. The authors focused on studying the thermal characteristics of the material in terms of thermal properties and crystallization behavior before and after printing. Based on their results, it was shown that there is no significant change in the thermal characteristics of the PA before and after printing. The 3D printed part's porosity is affected by the build orientation. Additionally, the results show that the porosity increases with the increase in distance from the printing bed. This was due to the large temperature gradient when printing far from the printing bed. This decrease in temperature limits the fusion process between printed beads and thus higher porosity is gained. Samples printed with $\mathrm{XZ}$ orientation showed better quasi-static flexural behavior than the XY; due to the increased porosity along the 
$\mathrm{Z}$ axis. Moreover, the $\mathrm{XZ}$ orientation shows higher overall fatigue life than the XY. The surface roughness has no significant effect on the fatigue behavior of the samples. Moreover, Terekhina et al. [67] conducted a comparison between FFF and selective laser sintering (SLS) 3D printing processes and the effect of the process on the flexure quasi-static and fatigue loading. In this study, PA was used as a model polymer. From their investigation, the FFF showed four times lower crystallinity of the produced samples, which caused a $16 \%$ decrease in the flexural stiffness. There was a difference in the porosity between the two 3D printing processes, as the FFF process produced parts with around $11 \%$ porosity. On the other hand, the surface roughness of the FFF process was around $43 \%$ higher. Despite these variations, there was no significant change in the flexural and fatigue properties of the produced samples.

Additionally, Fitzharris et al. [33] investigated the warpage that occurs during the printing of semicrystalline material. Polypropylene and polypropylene sulfide were selected as a model semicrystalline polymer. Material's characteristics such as coefficient of thermal expansion, thermal conductivity, heat capacity, and young's modulus were included in the simulation. Numerical simulation was used to simulate depositing a 2 to $10 \mathrm{~mm}$ long road of material on the printing platform with a constant temperature. It was found that the coefficient of thermal expansion is the main cause of warpage, however, thermal conductivity, heat capacity and young's modulus did not show a significant effect on the warpage phenomenon. Additionally, the large temperature gradient between the old deposited material and the freshly deposited one is one of the main reasons for this phenomenon.

Moreover, Armillotta et al. [68] provided an experimental and analytical model to overcome warpage defects on the processing material in the FFF system. This study investigated the behavior of these defects and characterized them on blockshaped parts in ABS thermoplastic resin as a function of various geometric variables, such as the thickness of deposited layers and the size of the processing part. The experimental results showed that thermal distortions (warpage) for a rectangular plate in ABS built by the FFF technique were mostly dependent on the maximum dimension of the horizontal plane and the length of a beam deflection under a uniform bending moment. Furthermore, increasing the layer thickness will have a moderate effect on the warpage, as a larger volume of material will be subjected to shrinkage during the thermal transient following the deposition of a new layer. Finally, different part shapes, such as a flat part with a complex profile may have a critical influence on the characteristic of the produced warpage.

Cattenone et al. [69] implemented a simulation model based on FE analysis to predict distortions in the FFF process. This model was tested with several parameters (e.g., material model, mesh size, and time step size) and was validated with experimental data. The result shows that the local temperature distribution, during the printing process, had a large influence on the time step size and a minor influence on mechanical performance. Also, choosing an appropriate meshing strategy has an important influence during the real printing process. The authors suggested having a finer meshing strategy for a small model, while a coarser meshing strategy for larger models (where large and small refers to the dimensions of the models compared with the filament dimensions). Lastly, the authors showed that the temperature dependence of yield stress limit and Young's modulus must be considered and calibrated to exert an acceptable result on the extruded filament and cannot be neglected when simulating an FFF process.

On the other hand, D'Amico and Peterson [70] provided a FE analysis model that is capable of simulating the heat transfer at sufficiently small time scales to capture the rapid cooling in the AM process. An experimental measurement was collected using a MatEx printer to validate the simulation results of the heat transfer obtained by the proposed model. The result indicates that high cooling rates with a common print speed may lead to larger residual stresses and reduced mechanical properties. By using a similar cooling profile, there will be a temperature deviation between the current and previous layers. It was also noticeable that the cooling rates showed a small dependence on a regular print speed $(10-30 \mathrm{~mm} / \mathrm{s})$ than for a higher print speed $(<30 \mathrm{~mm} / \mathrm{s})$, and the equilibrium with the environment temperature can be reached by sufficiently large parts. A maximum cooling rate and minimum time to reach the $\mathrm{Tg}$ can be observed between 10 and $30 \mathrm{~mm} / \mathrm{s}$ of print speeds and increasing time to $\mathrm{Tg}$ with higher print speeds. On the contrary, with high printing speed, the nozzle will move through each layer faster and start to deposit a new layer more rapidly, and this will raise effectively the steady-state temperature to which the layer is cooling.

\section{Conclusions and future perspective}

Various studies have explored the potential of transforming the developmental process of 3D printing technologies from the trial and error approach via experimentation into using virtual models based on the process properties and the material characteristics. Since the beginning of the development of the FFF 3D printing technology, many researchers have started to develop such models. This can be seen from the work by Bellini et al. [34], in which an analytical model was developed to describe the material flow and liquefier dynamics. In the meantime, other researchers worked on developing numerical models which allowed a better description of the material flow inside the printing head and also the behavior of the extrudates and eventually the printed part. An example of such an approach is evident in the research published by 
Serdeczny et al. [25, 71, 72]. Moreover, the availability of open-source hardware and software developed by the Reprap society after the expiration of the Stratasys FDM patents allowed researchers to monitor the process more successfully. This was shown by the work of Coogan and Kazmer $[73,74]$, in which a pressure sensor was installed in the printer nozzle which allowed on-line monitoring of the melt rheology and the material flow before extrusion. This enabled researchers to predict defects in printed parts using this on-line monitoring approach [75].

The future of the FFF 3D printing technology is promising as there are many researchers worldwide working on improving this technology; however, there are still many challenges. One of the major challenges is the material used to $3 \mathrm{D}$ print objects. Currently, many different materials are used that have very different rheological properties. These variations can be a limiting factor to the developed models as such models were developed for using just one or two materials. The approach of equipping the FFF technology with elements for on-line monitoring can be considered as a big move forward. Such tools are used for the prediction of defects. However, this step will enable researchers to investigate utilizing such elements for the production of closed-loop feedback systems. One of the challenges that need to be overcome in such a closed-loop system is the issue of over and under extrusion. As this problem is mainly caused by the melt rheology of the material being printed, such closed-loop systems can help to provide a suitable material flow during the 3D printing process. This approach, along with other monitoring systems that provide feedback for other elements of the 3D printing process, will help us to achieve a lower number of printing processes for products that have optimum quality.

Supplementary Information The online version contains supplementary material available at https://doi.org/10.1007/s00170-021-06918-6.

Code availability Not applicable.

Author contribution Conceptualization, methodology, investigation, and original draft preparation B.S., M.A., S.A., A.J., R.A., W.A. and M.Q.; review and editing, B.S., M.A., A.J., R.A. and P.C.; supervision, P.C., B.V.; project administration and funding acquisition, P.C. and B.V. All authors have read and agreed to the published version of the manuscript.

Funding This research was funded by the research project PRINTAID, the EU Framework Programme for Research and Innovation within Horizon 2020-MarieSklodowska-Curie Innovative Training Networks under grant agreement No. 722467.

Data availability Not applicable.

\section{Declarations}

Ethics approval and consent to participate Not applicable.
Consent for publication Approved.

Competing interests The authors declare no conflict of interest.

Open Access This article is licensed under a Creative Commons Attribution 4.0 International License, which permits use, sharing, adaptation, distribution and reproduction in any medium or format, as long as you give appropriate credit to the original author(s) and the source, provide a link to the Creative Commons licence, and indicate if changes were made. The images or other third party material in this article are included in the article's Creative Commons licence, unless indicated otherwise in a credit line to the material. If material is not included in the article's Creative Commons licence and your intended use is not permitted by statutory regulation or exceeds the permitted use, you will need to obtain permission directly from the copyright holder. To view a copy of this licence, visit http://creativecommons.org/licenses/by/4.0/.

\section{References}

1. Hull CW (1986) Apparatus for production of three-dimensional objects by stereolithography

2. Zhang Y, Shapiro V (2018)Linear-time thermal simulation of asmanufactured fused deposition modeling components. J Manuf Sci Eng 140:140. https://doi.org/10.1115/1.4039556

3. Cole DP, Riddick JC, Jaim HMI, Strawhecker KE, Zander NE (2016) Interfacial mechanical behavior of 3D printed ABS. J Appl Polym Sci 133. https://doi.org/10.1002/app.43671

4. Huang Y, Leu MC, Mazumder J, Donmez A (2015) Additive manufacturing: current state, future potential, gaps and needs, and recommendations. J Manuf Sci Eng 137. https://doi.org/10.1115/1. 4028725

5. Rejeski D, Zhao F, Huang Y (2018) Research needs and recommendations on environmental implications of additive manufacturing. Additive Manufacturing 19:21-28. https://doi.org/10.1016/j. addma.2017.10.019

6. Ngo TD, Kashani A, Imbalzano G, Nguyen KTQ, Hui D (2018) Additive manufacturing (3D printing): a review of materials, methods, applications and challenges. Compos Part B 143:172196. https://doi.org/10.1016/j.compositesb.2018.02.012

7. Singh S, Ramakrishna S, Singh R (2017) Material issues in additive manufacturing: a review. J Manuf Process 25:185-200. https://doi. org/10.1016/j.jmapro.2016.11.006

8. Stansbury JW, Idacavage MJ (2016) 3D printing with polymers: Challenges among expanding options and opportunities. Dent Mater 32:54-64. https://doi.org/10.1016/j.dental.2015.09.018

9. Tofail SAM, Koumoulos EP, Bandyopadhyay A, Bose S, O'Donoghue L, Charitidis C (2018) Additive manufacturing: scientific and technological challenges, market uptake and opportunities. Mater Today 21:22-37. https://doi.org/10.1016/j.mattod.2017. 07.001

10. Crump SS (1992) Apparatus and method for creating threedimensional objects

11. Schirmeister CG, Hees T, Licht EH, Mülhaupt R (2019) 3D printing of high density polyethylene by fused filament fabrication. Additive Manufacturing 28:152-159. https://doi.org/10.1016/j. addma.2019.05.003

12. Ning F, Cong W, Qiu J, Wei J, Wang S (2015) Additive manufacturing of carbon fiber reinforced thermoplastic composites using fused deposition modeling. Compos Part B 80:369-378. https://doi.org/10.1016/j.compositesb.2015.06.013 
13. Campbell I, Diegel O, Kowen J, Wohlers T (2018) Wohlers Report 2018: 3D printing and additive manufacturing state of the industry: Annual Worldwide Progress Report. Wohlers Associates

14. Chohan JS, Singh R (2017) Pre and post processing techniques to improve surface characteristics of FDM parts: a state of art review and future applications. Rapid Prototyp J 23:495-513. https://doi. org/10.1108/RPJ-05-2015-0059

15. Jaisingh Sheoran A, Kumar H (2020) Fused deposition modeling process parameters optimization and effect on mechanical properties and part quality: review and reflection on present research. Materials Today: Proceedings 21:1659-1672. https://doi.org/10. 1016/j.matpr.2019.11.296

16. Harris M, Potgieter J, Archer R, Arif KM (2019) Effect of material and process specific factors on the strength of printed parts in fused filament fabrication: a review of recent developments. Materials 12: 1664. https://doi.org/10.3390/ma12101664

17. Popescu D, Zapciu A, Amza C, Baciu F, Marinescu R (2018) FDM process parameters influence over the mechanical properties of polymer specimens: a review. Polym Test 69:157-166. https:// doi.org/10.1016/j.polymertesting.2018.05.020

18. Elkaseer A, Schneider S, Scholz SG (2020)Experiment-based process modeling and optimization for high-quality and resourceefficient FFF 3D printing. Appl Sci 10:2899. https://doi.org/10. 3390/app10082899

19. Shaqour B, Samaro A, Verleije B, Beyers K, Vervaet C, Cos P (2020) Production of drug delivery systems using fused filament fabrication: a systematic review. Pharmaceutics 12:517. https://doi. org/10.3390/pharmaceutics12060517

20. Abilgaziyev A, Kulzhan T, Raissov N, Ali MH, Match WLK, MirNasiri N (2015) Design and development of multi-nozzle extrusion system for 3D printer. In: 2015 International Conference on Informatics, Electronics Vision (ICIEV). pp 1-5

21. Aho J, Bøtker JP, Genina N, Edinger M, Arnfast L, Rantanen J (2019) Roadmap to 3D-printed oral pharmaceutical dosage forms: feedstock filament properties and characterization for fused deposition modeling. JPharmSci 108:26-35. https://doi.org/10.1016/j. xphs.2018.11.012

22. S. Henry, A. Samaro, F.H. Marchesini, B. Shaqour, J. Macedo, V. Vanhoorne, C. Vervaet, (2021)Extrusion-based 3D printing of oral solid dosage forms: Material requirements and equipment dependencies. International Journal of Pharmaceutics 598:120361. https://doi.org/10.1016/j.ijpharm.2021.120361

23. Parandoush P, Lin D (2017) A review on additive manufacturing of polymer-fiber composites. Compos Struct 182:36-53. https://doi. org/10.1016/j.compstruct.2017.08.088

24. Jiang Z, Diggle B, Tan ML, Viktorova J, Bennett CW, Connal LA (2020) Extrusion 3D printing of polymeric materials with advanced properties. Adv Sci 7:2001379. https://doi.org/10.1002/advs. 202001379

25. Serdeczny MP, Comminal R, Pedersen DB, Spangenberg J (2020) Experimental and analytical study of the polymer melt flow through the hot-end in material extrusion additive manufacturing. Additive Manufacturing 32:100997. https://doi.org/10.1016/j.addma.2019. 100997

26. Górecka Ż, Idaszek J, Kołbuk D, Choińska E, Chlanda A, Święszkowski W (2020) The effect of diameter of fibre on formation of hydrogen bonds and mechanical properties of 3D-printed PCL. Mater Sci Eng C 114:111072. https://doi.org/10.1016/j.msec. 2020.111072

27. Duty C, Ajinjeru C, Kishore V, Compton B, Hmeidat N, Chen X, Liu P, Hassen AA, Lindahl J, Kunc V (2018) What makes a material printable? A viscoelastic model for extrusion-based 3D printing of polymers. J Manuf Process 35:526-537. https://doi.org/10.1016/ j.jmapro.2018.08.008

28. Scheithauer U, Schwarzer E, Richter H-J, Moritz T (2015) Thermoplastic 3D printing — an additive manufacturing method for producing dense ceramics. Int J Appl Ceram Technol 12:2631. https://doi.org/10.1111/ijac.12306

29. Seppala JE, Migler KD (2016) Infrared thermography of welding zones produced by polymer extrusion additive manufacturing. Additive Manufacturing 12:71-76. https://doi.org/10.1016/j. addma.2016.06.007

30. Kendall K (2002) Chapter 3 - Energy analysis of adhesion. In: Dillard DA, Pocius AV, Chaudhury M (eds) Adhesion Science and Engineering. Elsevier Science B.V, Amsterdam, pp 77-110

31. McIlroy C, Graham RS (2018) Modelling flow-enhanced crystallisation during fused filament fabrication of semicrystalline polymer melts. Additive Manufacturing 24:323-340. https://doi.org/10.1016/j.addma.2018.10.018

32. Kim JY, Kim SY, Song YS, Youn JR (2011) Relationship between the crystallization behavior and the warpage of film-insert-molded parts. J Appl Polym Sci 120:1539-1546. https://doi.org/10.1002/ app. 33076

33. Fitzharris ER, Watanabe N, Rosen DW, Shofner ML (2018) Effects of material properties on warpage in fused deposition modeling parts. Int J Adv Manuf Technol 95:2059-2070. https://doi.org/10. 1007/s00170-017-1340-8

34. Bellini A, Güc, eri S, Bertoldi M (2004) Liquefier dynamics in fused deposition. J Manuf Sci Eng 126:237-246. https://doi.org/ 10.1115/1.1688377

35. Ramanath HS, Chua CK, Leong KF, Shah KD (2008) Melt flow behaviour of poly- $\varepsilon$-caprolactone in fused deposition modelling. J Mater Sci Mater Med 19:2541-2550. https://doi.org/10.1007/ s10856-007-3203-6

36. Mostafa N, Syed HM, Igor S, Andrew G (2009) A study of melt flow analysis of an ABS-iron composite in fused deposition modelling process. Tsinghua Sci Technol 14:29-37. https://doi.org/10. 1016/S1007-0214(09)70063-X

37. Monzón MD, Gibson I, Benítez AN, Lorenzo L, Hernández PM, Marrero MD (2013) Process and material behavior modeling for a new design of micro-additive fused deposition. Int J Adv Manuf Technol 67:2717-2726. https://doi.org/10.1007/s00170-012-4686y

38. Wittbrodt BT, Glover AG, Laureto J, Anzalone GC, Oppliger D, Irwin JL, Pearce JM (2013)Life-cycle economic analysis of distributed manufacturing with open-source 3-D printers. Mechatronics 23:713-726. https://doi.org/10.1016/j.mechatronics.2013.06.002

39. Ortega Z, Alemán ME, Benítez AN, Monzón MD (2016)Theoretical-experimental evaluation of different biomaterials for parts obtaining by fused deposition modeling. Measurement 89:137-144. https://doi.org/10.1016/j. measurement.2016.03.061

40. Anderegg DA, Bryant HA, Ruffin DC, Skrip SM, Fallon JJ, Gilmer EL, Bortner MJ (2019)In-situ monitoring of polymer flow temperature and pressure in extrusion based additive manufacturing. Additive Manufacturing 26:76-83. https://doi.org/10.1016/j. addma.2019.01.002

41. Tlegenov Y, Hong GS, Lu WF (2018) Nozzle condition monitoring in 3D printing. Robot Comput Integr Manuf 54:45-55. https://doi. org/10.1016/j.rcim.2018.05.010

42. Peng F, Vogt BD, Cakmak M (2018) Complex flow and temperature history during melt extrusion in material extrusion additive manufacturing. Additive Manufacturing 22:197-206. https://doi. org/10.1016/j.addma.2018.05.015

43. Shadvar N, Foroozmehr E, Badrossamay M, Amouhadi I, Dindarloo AS (2019) Computational analysis of the extrusion process of fused deposition modeling of acrylonitrile-butadiene-styrene. Int J Mater Form 14:121-131. https://doi.org/10.1007/ s12289-019-01523-1

44. Jerez-Mesa R, Travieso-Rodriguez JA, Corbella X, Busqué R, Gomez-Gras G (2016) Finite element analysis of the thermal 
behavior of a RepRap 3D printer liquefier. Mechatronics 36:119126. https://doi.org/10.1016/j.mechatronics.2016.04.007

45. Yang D, Wu K, Wan L, Sheng Y (2017) A particle element approach for modelling the $3 \mathrm{D}$ printing process of fibre reinforced polymer composites. Journal of Manufacturing and Materials Processing 1:10. https://doi.org/10.3390/jmmp1010010

46. Heller BP, Smith DE, Jack DA (2016) Effects of extrudate swell and nozzle geometry on fiber orientation in Fused Filament Fabrication nozzle flow. Additive Manufacturing 12:252-264. https://doi.org/10.1016/j.addma.2016.06.005

47. Mendes R, Fanzio P, Campo-Deaño L, Galindo-Rosales FJ (2019) Microfluidics as a platform for the analysis of 3D printing problems. Materials 12:2839. https://doi.org/10.3390/ma12172839

48. Li L, Sun Q, Bellehumeur C, Gu P (2002) Composite modeling and analysis for fabrication of FDM prototypes with locally controlled properties. J Manuf Process 4:129-141. https://doi.org/10.1016/ S1526-6125(02)70139-4

49. Zhang Y, Chou YK (2006)Three-dimensional finite element analysis simulations of the fused deposition modelling process. Proc Inst Mech Eng B J Eng Manuf 220:1663-1671. https://doi.org/10. 1243/09544054JEM572

50. Bellehumeur C, Li L, Sun Q, Gu P (2004) Modeling of bond formation between polymer filaments in the fused deposition modeling process. J Manuf Process 6:170-178. https://doi.org/10.1016/ S1526-6125(04)70071-7

51. Costa SF, Duarte FM, Covas JA (2008) Towards modelling of Free Form Extrusion: analytical solution of transient heat transfer. Int $\mathrm{J}$ Mater Form 1:703-706. https://doi.org/10.1007/s12289-008-0312-9

52. Xia H, Lu J, Dabiri S, Tryggvason G (2018) Fully resolved numerical simulations of fused deposition modeling. Part I: fluid flow. Rapid Prototyp J 24:463-476. https://doi.org/10.1108/RPJ-122016-0217

53. Zhang J, Wang XZ, Yu WW, Deng YH (2017) Numerical investigation of the influence of process conditions on the temperature variation in fused deposition modeling. Mater Des 130:59-68. https://doi.org/10.1016/j.matdes.2017.05.040

54. Liu J, Anderson KL, Sridhar N (2018) Direct simulation of polymer fused deposition modeling (fdm) - an implementation of the multi-phase viscoelastic solver in OpenFOAM. Int J Comput Methods 17:1844002. https://doi.org/10.1142/ S0219876218440024

55. Quinzani LM, Armstrong RC, Brown RA (1994) Birefringence and laser-Doppler velocimetry (LDV) studies of viscoelastic flow through a planar contraction. J Non-Newtonian Fluid Mech 52:136. https://doi.org/10.1016/0377-0257(94)85056-9

56. Xia H, Lu J, Tryggvason G (2019) Simulations of fused filament fabrication using a front tracking method. Int J Heat Mass Transf 138: 1310-1319. https://doi.org/10.1016/j.ijheatmasstransfer.2019.04.132

57. Bakrani Balani S, Chabert F, Nassiet V, Cantarel A (2019) Influence of printing parameters on the stability of deposited beads in fused filament fabrication of poly(lactic) acid. Additive Manufacturing 25:112-121. https://doi.org/10.1016/j.addma. 2018.10.012

58. El Moumen A, Tarfaoui M, Lafdi K (2019) Modelling of the temperature and residual stress fields during $3 \mathrm{D}$ printing of polymer composites. Int J Adv Manuf Technol 104:1661-1676. https://doi. org/10.1007/s00170-019-03965-y

59. Costa SF, Duarte FM, Covas JA (2017) Estimation of filament temperature and adhesion development in fused deposition techniques. J Mater Process Technol 245:167-179. https://doi.org/10. 1016/j.jmatprotec.2017.02.026

60. Coogan TJ, Kazmer DO (2017) Healing simulation for bond strength prediction of FDM. Rapid Prototyp J 23:551-561. https://doi.org/10.1108/RPJ-03-2016-0051
61. Fonseca J, Ferreira IA, de Moura MFSF, Machado M, Alves JL (2019) Study of the interlaminar fracture under mode I loading on FFF printed parts. Compos Struct 214:316-324. https://doi.org/10. 1016/j.compstruct.2019.02.005

62. Kallel A, Koutiri I, Babaeitorkamani E, Khavandi A, Tamizifar M, Shirinbayan M, Tcharkhtchi A (2019) Study of bonding formation between the filaments of PLA in FFF Process. IPP 34:434-444. https://doi.org/10.3139/217.3718

63. Northcutt LA, Orski SV, Migler KB, Kotula AP (2018) Effect of processing conditions on crystallization kinetics during materials extrusion additive manufacturing. Polymer 154:182-187. https:// doi.org/10.1016/j.polymer.2018.09.018

64. Vaes D, Coppens M, Goderis B, Zoetelief W, Van Puyvelde P (2019) Assessment of crystallinity development during fused filament fabrication through fast scanning chip calorimetry. Appl Sci 9: 2676. https://doi.org/10.3390/app9132676

65. Xinhua L, Shengpeng L, Zhou L, Xianhua Z, Xiaohu C, Zhongbin W (2015) An investigation on distortion of PLA thin-plate part in the FDM process. Int J Adv Manuf Technol 79:1117-1126. https:// doi.org/10.1007/s00170-015-6893-9

66. Terekhina S, Tarasova T, Egorov S, Skornyakov I, Guillaumat L, Hattali ML (2020) The effect of build orientation on both flexural quasi-static and fatigue behaviours of filament deposited PA6 polymer. Int J Fatigue 140:105825. https://doi.org/10.1016/j.ijfatigue. 2020.105825

67. Terekhina S, Tarasova T, Egorov S, Guillaumat L, Hattali ML (2020) On the difference in material structure and fatigue properties of polyamide specimens produced by fused filament fabrication and selective laser sintering. Int J Adv Manuf Technol 111:93-107. https://doi.org/10.1007/s00170-020-06026-x

68. Armillotta A, Bellotti M, Cavallaro M (2018) Warpage of FDM parts: experimental tests and analytic model. Robot Comput Integr Manuf 50: 140-152. https://doi.org/10.1016/j.rcim.2017.09.007

69. Cattenone A, Morganti S, Alaimo G, Auricchio F (2018) Finite element analysis of additive manufacturing based on fused deposition modeling: distortions prediction and comparison with experimental data. Journal of Manufacturing Science and Engineering 141:141. https://doi.org/10.1115/1.4041626

70. D'Amico A, Peterson AM (2018) An adaptable FEA simulation of material extrusion additive manufacturing heat transfer in $3 \mathrm{D}$. Additive Manufacturing 21:422-430. https://doi.org/10.1016/j. addma.2018.02.021

71. Serdeczny MP, Comminal R, Mollah MT, Pedersen DB, Spangenberg J (2020) Numerical modeling of the polymer flow through the hot-end in filament-based material extrusion additive manufacturing. Additive Manufacturing 36:101454. https://doi. org/10.1016/j.addma.2020.101454

72. Serdeczny MP, Comminal R, Pedersen DB, Spangenberg J (2019) Numerical simulations of the mesostructure formation in material extrusion additive manufacturing. Additive Manufacturing 28:419429. https://doi.org/10.1016/j.addma.2019.05.024

73. Coogan TJ, Kazmer DO (2018)In-line rheological monitoring of fused deposition modeling. J Rheol 63:141-155. https://doi.org/ 10.1122/1.5054648

74. Coogan TJ, Kazmer DO (2019) Modeling of interlayer contact and contact pressure during fused filament fabrication. J Rheol 63:655672. https://doi.org/10.1122/1.5093033

75. Coogan TJ, Kazmer DO (2020) Prediction of interlayer strength in material extrusion additive manufacturing. Additive Manufacturing 35:101368. https://doi.org/10.1016/j.addma.2020.101368

Publisher's note Springer Nature remains neutral with regard to jurisdictional claims in published maps and institutional affiliations. 